\title{
Consecuencias de la ausencia de regulación del financiamiento por terceros en el arbitraje comercial en Ecuador
}

\section{Consequences of the absence of regulation of third party funding in commercial arbitration in Ecuador}

\section{Daniel Fernando Ibarra*}

Recibido / Received: 07/05/2021

Aceptado / Accepted: 24/10/2021

DOI: $10.18272 /$ ulr.v8i2.2272

\section{Citación:}

Ibarra Villacís, D.F. «Consecuencias de la ausencia de regulación del financiamiento por terceros en el arbitraje comercial en Ecuador». USFQ Law Review, Vol 8, no 2, octubre de 2021, pp. 105-129, doi: $10.18272 /$ ulr.v8i2.2272. 


\section{RESUMEN}

Es un hecho que no hay regulación expresa respecto del TPF (financiamiento por terceros) en Ecuador y en varios países del mundo. Esta ausencia ha generado que varios expertos del arbitraje planteen problemas que pueden presentarse al utilizar este método. Por otro lado, existe otra corriente que propone que establecer una regulación estricta, limitaría el desarrollo del TPF. Sin embargo, tras analizar las posturas y problemas que respectan al TPF, se propone que sería más beneficioso y eficiente regular el TPF en los reglamentos de cada centro de arbitraje, a través de recomendaciones generales que no establezcan demasiadas barreras al funcionamiento de este método. El Ecuador tiene un ordenamiento jurídico amigable con el TPF, dado que este método no es contrario al orden público, y las partes pueden actuar bajo el principio de la autonomía de la voluntad ante la ausencia de prohibición expresa.

\section{Palabras Clave}

Arbitraje; financiamiento; financista; método; intereses

\section{Abstract}

It is a fact that there is no express regulation regarding the TPF in Ecuador and in several countries of the world. This absence has led to various arbitration experts raising issues that may arise when using this method. On the other hand, there is another current that proposes that establishing a strict regulation would limit the development of the TPF. However, after analyzing the positions and problems regarding the TPF, this paper proposes that it would be more beneficial and efficient to regulate the TPF in the regulations of each arbitration center, through general recommendations that do not establish too many barriers to the operation of this method. Ecuador has a legal system that is friendly to the TPF, since this method is not contrary to the public order, and the parties can act under the principle of autonomy of the will in the absence of an express prohibition.

\section{KEYWORDS}

Arbitration; champerty and maintenance; financing; funder; method; interest 


\section{INTRODUCCIÓN}

Third Party Funding ${ }^{1}$ o financiamiento por terceros, es un método utilizado en el arbitraje, al cual pueden acudir las partes con el objetivo de que un tercero, ajeno al conflicto, financie todos los costos del proceso arbitral; a cambio de un beneficio económico. ${ }^{2}$ Este tercero financista puede ser una compañía que busque invertir, o puede ser el estudio jurídico de abogados que representa a la parte en el arbitraje; no existe una limitación de quien puede ser financista. ${ }^{3}$ El financiamiento se maneja bajo un acuerdo entre las partes, un convenio de carácter privado y confidencial, que es completamente ajeno al conflicto objeto del arbitraje. Santiago Theoduloz, comenta respecto a la naturaleza del TPF, lo siguiente:

Así, vamos a estar frente a un TPF cuando; (i) Un tercero ajeno a las partes; (ii) soporte parte o la totalidad de los gastos (costos y honorarios) de un arbitraje, asumiendo en definitiva los riesgos de la actividad arbitral; (iii) con un determinado interés en la disputa que le reportará un beneficio. ${ }^{4}$

El financiamiento por terceros en el arbitraje es un método que puede llegar a abrir muchas puertas, pues no todos los interesados pueden acceder al mismo debido a sus elevados costos. ${ }^{5}$ Lisa Bench señala que el TPF es la solución para aquellas partes que no pueden acceder a un arbitraje por falta de recursos económicos; por lo que el financiamiento lógicamente abre las puertas a aquellos que de otra manera no podrían continuar con un reclamo arbitral, o ayuda a esos clientes con muchos reclamos en curso, mitigando su exposición al riesgo. ${ }^{6}$

Antes, muchos países anglosajones prohibían el financiamiento en litigios, y a pesar de que hoy en día han levantado esta prohibición, han guardado un silencio en cuanto al financiamiento en el arbitraje. ${ }^{7}$ Es importante analizar las consecuencias de la ausencia de regulación del TPF a nivel nacional e internacional; ya que se genera la duda de si podría convertirse en un problema para

1 En adelante TPF, por sus siglas en inglés.

2 Ver. Santiago Theoduloz, "Third Party Funding'; su relevancia e influencia actual en el mundo del arbitraje internacional”, Revista de Derecho 20 (2019): 159-187, https://doi.org/10.22235/rd.vi20.1850.

3 Bullard Falla Ezcurra, "¿Cómo Financiar Tu Arbitraje? Las Ventajas del Third Party Funding”, (Junio 2020). https://www. bullardabogados.pe/boletines/2020/Boletin_especial/day04-06/contenido.html\#pregunta1.

4 Theoduloz, "'Third Party Funding'; relevancia internacional," 164.

5 Ver. Noor Kadhim. "Whose Line of Credit is it Anyway? Third Party Funding Issues in Arbitration". Kluwer Arbitration Blog. (Julio 2017). http://arbitrationblog.kluwerarbitration.com/2017/07/17/whose-line-credit-anyway-third-party-funding-issues-arbitration/.

6 Traducción Libre, Lisa Bench Nieuwveld. "Third Party Funding - Investment of the Future?”. Kluwer Arbitration Blog. (Noviembre 2011). http://arbitrationblog.kluwerarbitration.com/2011/11/01/third-party-funding-investment-of-the-future/.

7 Harvard Law School Center on the Legal Profession, "A Brief History of Litigation Finance - The cases of Australia and the United Kingdom", Litigation Finance 5, no. 6 (Septiembre/Octubre 2019), https://thepractice.law.harvard.edu/article/a-brief-history-of-litigation-finance/. 
el arbitraje, o, por el contrario, si este silencio normativo permite una mayor libertad de acción para las partes.

Este artículo se centra en el arbitraje comercial en el Ecuador, tanto doméstico como internacional. A través de un análisis deductivo, se investigaron los principios del arbitraje y cómo los mismos permiten el desarrollo del TPF. También se revisaron casos de arbitraje donde la intervención de un financista ha sido cuestionada. Se consideró legislación comparada con países que han regulado el TPF; para luego aterrizar el análisis en el ordenamiento jurídico ecuatoriano y definir si Ecuador es un país que lo permite o lo limita y, finalmente, se presentan recomendaciones para mejorar la práctica del TPF en Ecuador.

\section{Situación actual del TPF}

Han pasado varios ańos desde que se empezó a aplicar este método de financiamiento en el arbitraje internacional, pero pocos países lo han regulado dentro de sus ordenamientos internos. ${ }^{8}$ Surgen distintas posiciones respecto al TPF, mismas que parten del hecho de que no existe regulación ni normativa internacional respecto de este tema.

Por ejemplo, Kiera Gans y Harout Samra señalan que si bien la actitud de esperar y ver pudo haber tenido sentido hace unos años, cuando evitar reaccionar exageradamente al fenómeno del TPF era totalmente comprensible; pero el rápido y persistente aumento de TPF en la comunidad de arbitraje sugiere que llegó el momento de actuar al respecto. ${ }^{9}$

Dentro de esta tendencia formalista, los doctrinarios que la respaldan plantean que debe existir una mayor regulación para garantizar seguridad jurídica. Francisco Blavi comentó sobre este punto que, "es importante apoyar el desarrollo de TPF y aprovechar sus beneficios, al mismo tiempo que se regula lo necesario para evitar incertidumbres y limitar sus peligros". ${ }^{10}$

Otro grupo de autores defienden la postura de que no se debe regular a fondo este método, ya que con los principios básicos y directrices generales del arbitraje internacional basta para establecer límites a este financiamiento. ${ }^{11}$ Ade-

8 International Council for Commercial Arbitration y Queen Mary University of London, Report of the Icca-Queen Mary Task Force on Third-Party Funding in International Arbitration, Reporte No. 4, abril de 2018.

9 Traducción Libre, Kiera Gans y Haroit J. Samra. "Chevron-Lago Agrio: the risks of third-party funding in multijurisdictional disputes” DLA PIPER. (Diciembre 2014). https://www.dlapiper.com/en/us/insights/publications/2014/12/international-arbitration-newsletter-q4-2014/chevron-lago-agrio/.

10 Traducción Libre, Francisco Blavi. "It's about time to regulate third party funding", Kluwer Arbitration Blog. (Diciembre 2015). http://arbitrationblog.kluwerarbitration.com/2015/12/17/its-about-time-to-regulate-third-party-funding/.

11 Marc Krestin y Rebecca Mulder. “Third-Party Funding In International Arbitration: To Regulate Or Not To Regulate?”. Kluwer Arbitration Blog. (Diciembre 2017). http://arbitrationblog.kluwerarbitration.com/2017/12/12/third-party-funding-international-arbitration-regulate-not-regulate/. 
más, sostienen que una mayor regulación en el tema limitaría la autonomía de la voluntad de las partes y la flexibilidad dentro del arbitraje. ${ }^{12} \mathrm{Al}$ respecto, Marc Krestin y Rebecca Mulder plantearon que, si bien el financiamiento por terceros es un área que requiere mayor regulación, intentar regularlo -al menos a nivel nacional- puede no necesariamente ser el mejor camino a seguir. ${ }^{13}$

Otra postura, apartada de las anteriores, se inclina por el ser más allá del deber ser, misma que señala que sería muy difícil regular el TPF por la infinidad de situaciones que podrían llegar a surgir en la práctica, por lo que recaerá en los tribunales y sus árbitros el deber de plantear los problemas legales que acarrea este método. ${ }^{14}$ Así como lo señalan Marc Krestin y Rebecca Mulder,

[...] es prácticamente imposible abordar todos los problemas y preocupaciones con un solo conjunto de reglas claras y vinculantes; Los problemas asociados con la financiación de terceros pueden diferir de un caso a otro, de una jurisdicción a otra y están destinados a cambiar con el tiempo, al igual que la forma en que se practica la financiación de terceros y la forma en que se percibe. No existe una 'talla única' y la flexibilidad es clave. ${ }^{15}$

Sin embargo, estas son solamente posturas doctrinarias respecto del financiamiento por terceros. Para poder llegar a una conclusión o poder realizar una propuesta sustentada, el trabajo también analizará casos reales de arbitrajes financiados con el fin de entender cómo funciona este método actualmente en la práctica, sin una regulación clara.

\section{Desarrollo}

\subsection{ConseCUENCIAS QUE aCARREa La AUSENCIA DE REgUlaCión DEL TPF}

\subsubsection{Problemas doctrinarios}

Existen varios autores, árbitros y abogados, dentro del mundo del arbitraje, que han criticado el uso del TPF, planteando inconvenientes que podría acarrear este método de financiamiento. ${ }^{16}$ Críticas que deben ser revisadas para

12 Traducción Libre, Charlie Morris y Franca Ciambella. "Arbitration and Litigation Funding in Singapore and Hong Kong", Woodsford Litigation Funding. (Junio 2020). https://woodsfordlitigationfunding.com/white-paper-arbitration-litigation-funding-singapore-and-hong-kong2/\#:-:text=Since\%20Singapore's $\% 20$ Parliament $\% 20$ passed $\% 20$ the, associated $\% 20$ proceedings $\% 20$ (for $\% 20$ example $\% 2 \mathrm{C} \% 20$ enforcement.

13 Krestin y Mulder. “TPF To Regulate Or Not?”.

14 Traducción Libre, Joseph J. Stroble y Laura Welikson, "Third-Party Litigation Funding: A Review of Recent Industry Developments", Defense Counsil Journal 87, no. 1 (2020): 1-18, https://www.iadclaw.org/assets/1/17/Third-Party_Litigation_Funding_-_A_Review_of_Recent_Industry_Developments2.pdf? 4180 .

15 Krestin y Mulder. "TPF To Regulate Or Not?".

16 International Council for Commercial Arbitration y Queen Mary University of London, Report Of The Icca-Queen Mary 
poder establecer si pudiesen ser superables o si son realmente barreras o problemas para el sistema arbitral.

Un evidente problema es el posible conflicto de intereses, ya que no se puede negar que habrá varios arbitrajes en los que exista un claro conflicto de intereses entre el árbitro y el financista, y esto se debe a que el arbitraje internacional tiene un número limitado de árbitros, abogados y partes, que ya se conocen entre sí. El arbitraje es una industria concentrada a la que, a pesar de ser internacional, las partes que recurren a este método de solución de conflictos suelen ser las mismas, por lo que existen ya estrechas relaciones entre ellas. Garino dice al respecto que,

Algunos autores argumentan que estos conflictos de interés podrían ser comunes, considerando que la industria del arbitraje internacional se encuentra altamente concentrada. Apuntan al incremento del uso de TPF; al reducido número de firmas financiadoras; a relación "simbiótica" entre financiadores y un pequeńo grupo de firmas de abogados; y los vínculos estrechos entre las firmas de élite y los principales árbitros internacionales. ${ }^{17}$

Ante esta situación se propone que, "sin duda revelar la existencia de un TPF es la herramienta que realmente permitirá conocer la existencia de cualquier tipo de conflicto de intereses" ${ }^{18}$ Una vez que se conoce del TPF, pueden tomarse como referencia las Directrices IBA (International Bar Association) de Conflictos de Intereses, y determinar si la relación realmente afectaría a la imparcialidad del árbitro. ${ }^{19}$

Cabe notar que no existe una regla general, dado que las Directrices IBA contienen una lista no taxativa de relaciones que pueden presentarse en los arbitrajes ${ }^{20}$ por lo que deberá analizarse cada caso en concreto. Este instrumento de soft law establece situaciones ante las cuales el árbitro está obligado a excusarse por la clara presencia de conflicto de intereses, pero también situaciones en las que, una vez revelados los hechos controvertidos, dependerá de las partes el acordar si el árbitro continúa en el proceso o si lo reemplazarán. ${ }^{21}$

Las Directrices IBA son un instrumento muy útil para resolver este problema, ya que una vez que la parte financiada revele el financiamiento, y las partes hayan analizado la relación de este tercero con el árbitro basado en las Directrices IBA; se derivará automáticamente a 2 situaciones: 1) el árbitro será

Task Force On Third-Party Funding In International Arbitration, Reports No. 4, abril de 2018.

17 Joaquín Garino Podestá y Sebastián Picardo González. "Acceso a la justicia, prácticas abusivas y el paraíso de los apostadores: Third Party Funding en la práctica arbitral internacional", Revista de Derecho de la Universidad de Montevideo 35, (2019), 39-60.

18 Theoduloz, "'Third Party Funding'; relevancia internacional," 85.

19 Aren Goldsmith y Lorenzo Melchionda, "The ICC's Guidance Note on Disclosure and Third-Party Funding: A Step in the Right Direction”. Kluwer Arbitration Blog, Marzo 14, 2016.

20 Directrices IBA.

21 Id. 
reemplazado, o 2) las partes han descartado cualquier conflicto de intereses y desean continuar con el mismo árbitro; evitando así cualquier problema de imparcialidad dentro del resto del proceso. ${ }^{22}$

Otra crítica al TPF es que podría existir un abuso del derecho por parte de los demandantes, y así lo estableció Joaquín Garino, quien sostiene que, "el TPF puede incentivar la presentación de demandas especulativas exageradas, o que no hubieran sido presentadas ante la falta de TPF". ${ }^{23}$

Si bien es cierto que con el apoyo de un financista habrá quienes comiencen arbitrajes que normalmente no lo harían si se tratase de utilizar sus propios fondos, es importante tomar en cuenta que los financistas realizan auditorias y una debida diligencia a los casos, con el fin de asegurar un beneficio, caso contrario no sería visto como una inversión. ${ }^{24}$ Es decir, los inversionistas no financiarían cualquier caso que no tenga fundamento suficiente ni posibilidad de éxito.

No existe un límite al financiamiento en un arbitraje, dado que las dos partes podrían ser financiadas, incluso por el mismo financista, ${ }^{25}$ y esto podría generar arbitrajes simulados y otras prácticas abusivas, convirtiendo al arbitraje internacional en un centro de apuestas. ${ }^{26}$ Sin embargo, ante este problema, Theoduloz estableció que,

Ahora, ¿existe el riesgo de que una creciente industria de financiamientos de arbitraje genere procesos arbitrales por los beneficios económicos que la actividad conlleva? Entendemos que la única forma de que esto no suceda es regulando a los TPF, pero de ninguna manera prohibiendo los mismos. ${ }^{27}$

Otro problema se plantea respecto a las costas, donde una de las partes no solamente solicita que la parte perdedora cubra sus propios costos, sino también los de la parte que ha prevalecido en el arbitraje. La problemática se refleja en lo comentado por Carlos Ríos: "La lógica detrás de ello suele ser simple: si no hay dinero para cubrir los propios gastos, mucho menos habrá para reembolsar aquellos en los que ha incurrido la contraparte". ${ }^{28}$ Sin embargo, la presencia del tercero financista cambia esta situación, dado que es alguien que tiene suficientes recursos para cubrir los costos del arbitraje. ${ }^{29}$

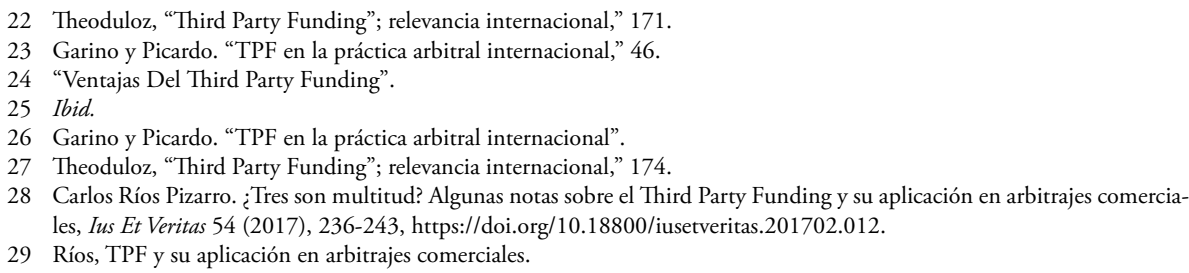


Otro problema relacionado que surge es hasta qué punto puede el tribunal obligar o actuar sobre el financista, de qué manera el árbitro tiene potestad sobre el financista, si el mismo es un tercero ajeno al conflicto, a quien no se le aplicarían las reglas o leyes del arbitraje. ${ }^{30} \mathrm{Al}$ respecto Garino plantea lo siguiente:

[...] el TPF puede exponer a la parte demandada a lo que la doctrina llama ' hitand-run' arbitral. Se conoce como arbitrajes 'hit-and-run' a aquellos en los cuales la parte demandante insolvente -valiéndose de TPF- no tiene éxito en la disputa, pero ni la demandante ni el tercero financiador se hacen cargo de una condena adversa en costos el 'hit-and-run' requiere que el tercero no haya asumido la responsabilidad por estos costos en el acuerdo de financiamiento respectivo. ${ }^{31}$

Una vez analizados los problemas propuestos por aquellos críticos del TPF, se vio que los mismos pueden resolverse a través de otros métodos que no implican necesariamente una regulación estricta. La naturaleza misma del TPF ayuda a evitar ciertos problemas, por ejemplo, evitar el abuso del derecho a través auditorias que analicen el caso antes del financiamiento; o los principios y costumbres de la comunidad de arbitraje, que proponen solventar el conflicto de intereses a través de las Directrices IBA. Se puede notar que a pesar de no existir regulación expresa y directa sobre el TPF, los problemas planteados pueden ser superables sin la necesidad de una normativa estricta.

\subsubsection{Problemas recopilados por el Consejo Internacional de Arbitraje Comercial}

El Consejo Internacional de Arbitraje Comercial ${ }^{32}$ es una organización dedicada a promover el uso y mejorar los procesos de arbitraje, y la Universidad Queen Mary de Londres es una de las principales universidades centradas en la investigación del Derecho durante más de 130 años. El Task Force, formado por estas dos instituciones, está compuesto por una amplia gama de profesionales y líderes expertos: árbitros, abogados, firmas, empresas, instituciones arbitrales, académicos y una variedad de terceros financiadores. Su objetivo era realizar un informe en el que se planteara todo lo relevante que respecte al TPF, desde su experiencia. ${ }^{33}$

El Grupo de Trabajo del ICCA-Queen Mary sobre el Financiamiento por Terceros en el Arbitraje Internacional ${ }^{34}$ planteó una serie de problemas con el TPF, entre los que señaló que será cuestión de tiempo que surjan casos en los

30 Report of the Icca-Queen Mary Task Force On Third-Party Funding In International Arbitration.

31 Garino y Picardo. "TPF en la práctica arbitral internacional," 46.

32 En adelante ICCA por sus siglas en inglés, International Council For Commercial Arbitration.

33 International Council for Commercial Arbitration y Queen Mary University of London, Report Of The Icca-Queen Mary Task Force On Third-Party Funding In International Arbitration, Reports No.4, abril de 2018.

34 En adelante ICCA-QM, por sus siglas en inglés, International Council For Commercial Arbitration-Queen Mary. 
que se dé un conflicto de intereses debido a la concentración que existe en este mercado. $\mathrm{Al}$ respecto han dicho que:

Otros factores incluyen el creciente número de casos que involucran financiamiento de terceros, el segmento altamente concentrado de la industria del financiamiento que invierte en casos de arbitraje internacional, la relación simbiótica entre los financiadores y un pequeño grupo de firmas de abogados. ${ }^{35}$

Debido al reducido número de firmas de abogados y de financistas que trabajan en este mercado actualmente, tarde o temprano llegará a generarse algún tipo de relación entre las partes y los árbitros, o las partes y los financistas, o los financistas y los árbitros; ${ }^{36}$ tomando en cuenta el factor de que no existe una restricción o límite a quién puede ser un financista. ${ }^{37}$

Otro problema que surgió es el alcance que tendría la confidencialidad que caracteriza a los arbitrajes, y si esta cubrirá también al tercero financista, es decir, qué pasa con la información que el tercero financista aporte a su financiado, o qué pasa si el financista quiere saber el estado del caso y existe un claro acuerdo de confidencialidad en el que solo las partes podrán conocer sobre este. ${ }^{38}$ Mayor sería el problema en el que las partes acuerden que el laudo arbitral será confidencial solamente para ellos y el árbitro, ¿cómo podrá el tercero financista conocer sobre si le beneficia o no el laudo? Con base a esta investigación, el Grupo de Trabajo concluyó que,

En la mayoría de las jurisdicciones, no hay una respuesta clara sobre si los documentos y la información proporcionados a un financiador serán definitivamente protegidos, $[\ldots]$ no hay precedentes o reglas bien establecidos [...] con el punto. ${ }^{39}$

Al respecto, el Grupo de Trabajo de la ICCA-MQ, señaló que no se tiene claro todavía sobre si la confidencialidad alcanza a proteger también la información del tercero, como en los documentos que él aporte al caso, "existe una categoría separada (documentos legales creados internamente por el financiador), y es poco probable que estos documentos se usen en el arbitraje ya que el financiador no es parte del procedimiento". 40 Se ha llegado a cuestionar que, dado que el tercero no es en sí una parte del arbitraje, si los principios y reglas de este no deberían obligarlo, así como tampoco protegerlo. ${ }^{41}$

35 Traducción Libre, Report of the Icca-Queen Mary Task Force On Third-Party Funding In International Arbitration., párr. 82.

36 Id.

37 "Ventajas del Third Party Funding".

38 Ryan Smith, "'Tempering the Gambler's Nirvana' A Review into to the issues and regulation of Third Party Funding in Investment Treaty Arbitration”. Tesis de maestría. Uppsala University, 2018. http://www.diva-portal.org/smash/record. jsf?pid=diva2\%3A1212686\&dswid=7455.

39 Traducción libre, Report Of The Icca-Queen Mary Task Force On Third-Party Funding In International Arbitration, párr. 124.

40 Traducción Oficial, Id, párr, 119.

41 Francisco Blavi, "It's about time to regulate third party funding." Kluwer Arbitration Blog. (Diciembre 2015). http:// arbitrationblog.kluwerarbitration.com/2015/12/17/its-about-time-to-regulate-third-party-funding/. 
El Grupo de Trabajo también se pronunció respecto de las costas que podría solicitar una parte en el arbitraje. En caso de que el tribunal condene a la parte que está siendo financiada, surgió la siguiente duda: ¿podrá el tribunal obligar al financista a pagar estas costas a pesar de que el tercero no es una parte en el arbitraje? El Grupo de Trabajo no tiene clara una respuesta al respecto, pero se señaló que:

En el arbitraje comercial, en este momento no existe una tendencia uniforme para las aplicaciones de costos. Por lo tanto, los tribunales internacionales tendrán que decidir la garantía de las solicitudes de costos con base a las normas pertinentes de la legislación nacional aplicable. ${ }^{42}$

El Grupo de Trabajo de la ICCA-QM, es una entidad compuesta por los mayores expertos en TPF alrededor del mundo, pero incluso ellos se han limitado a enlistar una serie de problemas respecto del TPF, mas no a proponer una solución o una forma de regulación. Han establecido que no existen precedentes suficientes, ni factores objetivos sustentables que sirvan para proponer una regulación al TPF. Sin embargo, son problemas reales que aquellos países que promuevan la práctica de arbitrajes financiados, deben tomar en cuenta e intentar evitarlos.

\subsection{EL FINANCIAMIENTO POR TERCEROS EN LA PRÁCTICA: ANÁLISIS DE CASOS}

Debido al carácter reservado de algunos arbitrajes financiados, no se tiene mucha información pública al respecto de lo que ha sucedido en estos procesos, principalmente, porque aparte de la confidencialidad del arbitraje, existe confidencialidad en el acuerdo de financiación entre la parte y el tercero. ${ }^{43}$ Es importante señalar que la revisión de los siguientes casos se enfocó en las primeras etapas del arbitraje, no se analizaron laudos que establezcan parámetros vinculantes respecto del TPF.

Se revisó el caso entre Guaracachi America Inc., empresa inversionista de Estados Unidos, y Rurlec PLC, empresa inversionista del Reino Unido; ambas como partes demandantes y partes financiadas; contra el Estado de Bolivia, como demandado. Un arbitraje de inversiones que se desarrolló bajo Reglas UNCITRAL, el cual surgió a raíz de un tratado de inversiones entre Estados Unidos y Bolivia, y otro entre el Reino Unido y Bolivia. ${ }^{44}$

Dentro del arbitraje se conocía que los demandantes estaban siendo financiados, pero no se conocía quién era el financista. Una vez que terminó el litigio,

42 Report of the Icca-Queen Mary Task Force On Third-Party Funding In International Arbitration. párr. 165.

43 Ver. Goldsmith y Melchionda, "Disclosure and Third-Party Funding".

44 Caso No. 2011-17, Guaracachi America, Inc. and Rurelec PLC v. The Plurinational State of Bolivia, UNCITRAL, PCA. 
el financista reveló su identidad y el Estado boliviano declaró conocerlo, ya que la participación de este había generado conflictos procesales entre inversionistas y Estados anteriormente. De haber sabido la identidad del tercero antes, el Estado boliviano señaló que hubiese presentado un reclamo al respecto. ${ }^{45}$

Otro caso revisado fue el de Oxus Gold pic, parte demandante; y República de Uzbekistán, como parte demandada, proceso que se desarrolló bajo las reglas UNCITRAL. El tribunal notó claramente que el demandante estaba siendo financiado por un tercero y sentó razón en dos providencias del proceso para conocimiento de la parte demandada. ${ }^{46}$

El tribunal dijo que el financiamiento variará en cada caso y dependerá de cada tribunal cómo se maneje el arbitraje, dado que no existen precedentes sustantivos obligatorios con respecto al financiamiento por terceros. El tribunal estableció en el laudo que en este caso la financiación por parte del tercero no afectó en la esencia del proceso arbitral en ningún aspecto. ${ }^{47}$

En Canadá, la Corte Suprema estableció una decisión unánime en el caso entre 9354-9186 Québec inc. y 9354-9178 Québec inc., como demandantes; y Callidus Capital Corporation, como demandado y como parte financiada. Se trataba de un juicio en el que los demandados eran deudores; $y$ un tema relevante a tratar durante todo el caso fue si la Corte Suprema podía o no permitir que los demandados tengan financiamiento en el litigio. ${ }^{48}$

Se estableció que el TPF no era ilegal, pero que la legalidad de los acuerdos de financiación seguía siendo un tema en evolución. A raíz de esta decisión los arbitrajes internacionales con sede en Canadá están considerando el TPF. La posición de la Corte Suprema brinda más acceso a la justicia, y ahora la jurisdicción canadiense ofrece mayor seguridad a las partes, ya que el financiamiento no se considerará contrario al orden público en Canadá. ${ }^{49}$

Otro caso de Canadá es el caso de Houle v St Jude Medical Inc., ${ }^{50}$ en el que la Corte Superior de Ontario estableció que el aprobar o denegar la financiación dependerá de las circunstancias particulares de cada caso, pero que con base a la ley aplicable, el tribunal deberá satisfacer por lo menos estos 4 criterios para aprobar la financiación: (1) el acuerdo necesariamente deberá ser solo para permitir el acceso a la justicia; (2) el acceso a la justicia debe ser sustancialmente significativo; (3) el acuerdo debe ser justo y razonable, debe proteger

45 Prior IAReporter Coverage Of Guaracachi And Rurelec v. Bolivia, Reporte de fecha 3 de junio de 2014, se puede revisar los reportes de este caso de la IA en https://www.iareporter.com/arbitration-cases/guaracachi-and-rurelec-v-bolivia/.

46 Oxus Gold pic v Republic of Uzbekistan, UNCITRAL, Laudo final, 17 diciembre de 2015, párr. 127.

47 Prior IAReporter Coverage Of Oxus Gold v Uzbekistan, se pueden revisar los reportes de este caso de la IA en https://www. iareporter.com/arbitration-cases/oxus-gold-v-uzbekistan/.

48 Québec inc. v. Callidus Capital Corp., 2020 SCC 10 (CanLII), https://canlii.ca/t/j7c04.

49 Hugh Meighen, "The Third Party Litigation Funding Law Review: Canada", The Law Review (2021).

50 Houle v St. Jude Medical Inc. Corte Superior de Ontario. 
los verdaderos intereses de la parte; y (4) el financista no debe ser compensado en exceso, solo por haber asumido los riesgos. ${ }^{51}$

Un caso cercano a la jurisdicción ecuatoriana es el controversial caso de Chevron-Lago Agrio, en el que el Estado ecuatoriano es la parte accionante, y Chevron es la parte demandada y la parte financiada. ${ }^{52}$ La decisión de la Corte del Distrito Sur de Nueva York resaltó que existieron ciertas complicaciones envueltas en la financiación por terceros en las disputas multi jurisdiccionales, sin embargo, no se tiene conocimiento de qué clase de complicaciones, dado que la Corte ha guardado confidencialidad al respecto. ${ }^{53}$

En este caso, la parte financiada, Chevron, había tomado la decisión de no revelar información significativa del acuerdo de financiación dado que era confidencial. Solamente reveló que estaba siendo financiada pero no la identidad del financista; la Corte de NY tuvo problemas con esta no revelación ya que incluso hubo problemas al momento de pagar las tarifas del arbitraje. ${ }^{54}$ Problemas de los que no se conoce a fondo, por su confidencialidad.

No se encontró un patrón que se repita en los casos revisados en este trabajo, dado que en algunos casos el financiado fue la parte actora y en otros fue la parte demandada. En los casos analizados no se repitió ningún problema. Por ejemplo, en el caso de Bolivia se vio que el problema no era por el financiamiento en sí, sino por quién era el financista. Por otro lado, en el caso de Ecuador se vio que el problema era la falta de revelación del financiamiento, y la confidencialidad que guardó la parte financiada al respecto.

Sin embargo, también se vieron casos financiados sin inconvenientes. Por ejemplo, los casos de Canadá no presentaron problema alguno, incluso uno de ellos sirvió como precedente vinculante para levantar la prohibición del financiamiento en Canadá, estableciendo que no sería contrario al orden público. ${ }^{55}$ Tampoco se encontraron suficientes casos como para establecer conclusiones respecto de un problema que se repita siempre en arbitrajes financiados; solamente se realizó una breve revisión de casos a los cuales se tuvo acceso, para ver cómo se practica el TPF en casos reales y qué pronunciamientos hay sobre este método por parte de los árbitros en los mismos.

51 Meighen, "The TPF: Canada".

52 Chevron - Lago Agruio, Corte del Distrito Sur de Nueva York, 2014.

53 Gans y Samra, "Chevron: the risks of TPF".

54 Ibid.

55 Meighen, "The TPF: Canada". 


\subsection{Países Que Regulan el financiamiento por terceros}

Es relevante para el análisis del presente trabajo revisar las legislaciones de aquellos países que han comenzado a regular el TPF, para analizar de qué manera lo han hecho, si desde una tendencia formalista de establecer varios límites o incluso prohibiciones a este método de financiamiento, o si, por el contrario, se han enfocado en establecer directrices generales para un mejor funcionamiento del mismo. Esta revisión también servirá como guía para plantear soluciones a los problemas anteriormente señalados.

\subsubsection{Regulación en Singapur}

Singapur es uno de los países pioneros en regular el financiamiento por terceros dentro de su ordenamiento jurídico, ${ }^{56}$ específicamente a través de la enmienda añadida a su Código Civil en 2016, en la que levanta la prohibición al champerty y al maintenance, ${ }^{57}$ figuras de financiamiento clásicas del common law. ${ }^{58}$ Esta enmienda define el financiamiento, a quienes se los cataloga como financiadores y financiados, y dentro de qué método de solución de disputas será permitido el financiamiento por un tercero sin que sea contrario al orden público. Las definiciones textuales que tipifica esta norma son:

'Financiador externo' significa una persona que se ocupa de financiar la totalidad o parte de los costos de los procedimientos de resolución de disputas en los que la persona no es parte;

'Parte financiada' significa una parte en un procedimiento de resolución de disputas que celebra un acuerdo con un Tercero Financiador para el financiamiento de la totalidad o parte de los costos del procedimiento e incluye un liquidador o administrador judicial;

'Contrato de financiación de terceros' significa un contrato o acuerdo de una parte o parte potencial para un procedimiento de resolución de disputas con un tercero financiador para el financiamiento de la totalidad o parte de los costos del procedimiento a cambio de una participación u otro interés en el producto o producto potencial de los procedimientos a los que la parte o parte potencial pueda tener derecho

'Procedimiento de resolución de disputas' significa el proceso completo de resolver o intentar resolver una disputa entre 2 o más partes e incluye cualquier procedimiento civil, de mediación, conciliación, arbitraje o de insolvencia. ${ }^{59}$

56 Stroble y Welikson, "TPF: Industry Developments".

57 Sección 5A (1). Civil Law (Amendment), Bill No. 38/2016, de noviembre de 2016.

58 Petit, Sherina. "Maintenance and champerty: An end to historic rules preventing third-party funding?", Norton Rose Fulbright. (Septiembre 2016). https://www.nortonrosefulbright.com/en/knowledge/publications/bfofd6fe/maintenance-and-champerty.

59 Civil Law (10) (Amendment) Bill No. 38/2016. 
La enmienda al Código Civil de Singapur es una regulación estricta que pone límites y normas de funcionamiento del TPF, y señala que ante el incumplimiento de estas directrices, el financiamiento se considerará contrario al orden público. Sin embargo, sigue siendo muy general y deja en manos de los legisladores o ministros el generar los respectivos reglamentos para que esta enmienda tenga efecto. Específicamente en su artículo establece que:

8) El Ministro puede hacer que los reglamentos necesarios o convenientes sean prescritos para llevar a cabo o dar efecto a esta sección, incluyendo:

(a) prescribir las calificaciones y otros requisitos que un Tercero Financiador debe satisfacer o cumplir para ser un Tercero Financiador calificado. ${ }^{60}$

Los principales objetivos de esta enmienda fueron: 1) establecer requisitos para que un financista y financiado se puedan catalogar como tal, y aclara que si no se cumplen los mismos, el tercero no tendrá los derechos del acuerdo de financiación. No tipifica los requisitos necesarios, sino que 2) señala que los legisladores deberán hacer las enmiendas necesarias para establecer los requisitos para el financiamiento por terceros. ${ }^{61}$

\subsubsection{Regulación en Hong Kong}

Hong Kong es otro de los primeros países en regular el financiamiento por terceros en su ordenamiento jurídico a través de la Ley de Arbitraje y Mediación (Financiamiento por Terceros) (Enmienda) Ordenanza, 2017. Esta ley está compuesta por definiciones, requisitos, directrices, y también levanta la prohibición de maintenance y champerty. ${ }^{62}$ En su sección introductoria, esta ley establece que servirá "para garantizar que la financiación de terceros en el arbitraje o en mediación no esté prohibida por las doctrinas del common law de manutención y champerty; y proveer las medidas y salvaguardias necesarias". ${ }^{63}$

La Ley define el TPF y cada uno de sus elementos, también establece requisitos necesarios para que el acuerdo de financiamiento entre las partes sea válido ${ }^{64}$ En su capítulo 5 señala el tipo de comunicación que debe existir dentro de un arbitraje financiado, y la obligación de revelar: 1) que la parte está siendo financiada, 2) el nombre del financiador, y 3) cuándo termina la financiación. También indica que esta información debe revelarse a todas las partes del proceso, tanto árbitros como contraparte. El artículo -en su idioma original con el fin de mantener la esencia del mismo- señala lo siguiente:

$60 \quad I d .,(8)$

61 Civil Law (Amendment), Bill No. 38/2016. Explanatory Statement.

62 Division 3. 98 L. Arbitration and Mediation Legislation (Third Party Funding) (Amendment) Ordinance No. 6 de 2017.

63 Arbitration and Mediation Legislation (Third Party Funding) (Amendment).

64 Id., Division 2. Interpretation. 
98U. Disclosure about third party funding of arbitration

(1) If a funding agreement is mad, the funded party must give written notice of

(a) the fact that a funding agreement has been made; and

(b) the name of the third party funder.

(3) The notice must be given to

(a) each other party to the arbitration; and

(b) the arbitration body. ${ }^{65}$

Esta regulación se limita a establecer recomendaciones generales, y no entra a enlistar taxativamente limitaciones o problemas. Esto podría ser beneficioso para el TPF, porque la regulación brinda libertad de acción a las partes, pero a la vez establece parámetros a nivel nacional para que todos los arbitrajes mantengan una misma línea. Al respecto, Woodsford, una de las empresas más grandes de financiamiento en litigación y arbitraje, ha señalado que:

Los cambios recientes en Hong Kong y Singapur han demostrado un compromiso con el principio de autonomía de las partes y 'acceso a la justicia' en el arbitraje y han elevado el perfil del sistema judicial en ambas jurisdicciones. ${ }^{66}$

\subsubsection{Regulación en Canadá}

Canadá es un país con una legislación de common law que prohibía el maintenance y champerty. Sin embargo, como ya se mencionó anteriormente, la Corte Suprema levantó esta prohibición y sentó razón de que el financiamiento en litigios y arbitraje no sería contrario al orden público canadiense. ${ }^{67}$ Aparte, Canadá suscribió un instrumento internacional con la Unión Europea, el Acuerdo Económico y Comercial Global, ${ }^{68}$ un acuerdo integral de economía y comercio entre Canadá y la Unión Europea, en el cual tratan el financiamiento por terceros.

El CETA comienza su capítulo 8, correspondiente a Inversión, definiendo conceptos que serán objetos de las relaciones comerciales del tratado, y define al TPF. Luego señala que la parte financiada debe revelar a la contraparte y al tribunal la identificación del financista. Indica que la etapa para revelar el financiamiento debe ser al presentar la demanda, y que la terminación se notificará el instante en el que concluya el acuerdo. De manera textual el artículo establece lo siguiente:

Artículo 8.26 - Financiamiento de terceros

1. Cuando exista financiamiento de un tercero, la parte contendiente que se beneficie del mismo revelará a la otra parte y al Tribunal el nombre y la identificación

$6598 \mathrm{U}$. Arbitration and Mediation Legislation (Third Party Funding) (Amendment)

66 Morris y Ciambella, "Arbitration Funding in Singapore and Hong Kong".

67 Houle v St. Jude Medical Inc. Corte Superior de Ontario.

68 En adelante CETA por sus siglas en inglés, Canada-European Union Comprehensive Economic and Trade Agreement. 
del tercero financiador.

2. La divulgación se hará al momento de la presentación de la demanda. Si el acuerdo de financiamiento concluye, o si el financiamiento se realiza después de la presentación de la demanda, la divulgación se hará tan pronto como se concluya el acuerdo o tan pronto se haga la donación. ${ }^{69}$

El CETA enfoca solamente un capítulo al TPF, por lo que todavía es un instrumento legal muy general, sin embargo, se aprecia que se limita a establecer recomendaciones y definiciones para que las partes tengan claros los conceptos dentro de un arbitraje financiado. No es una ley que establezca límites o prohibiciones; solamente establece directrices para un mejor funcionamiento del arbitraje.

Tras analizar estas tres legislaciones internacionales, se notó que la manera en la que han regulado el TPF no es muy estricta, pues se han limitado a establecer recomendaciones y definiciones. Un patrón que se repite es la obligación de revelación del financiamiento y del financista. Estas normas quieren evitar la existencia de cualquier conflicto de intereses en los arbitrajes financiados de sus países, para evitar que existan problemas con la figura del TPF.

\subsection{El TPF EN ECUADOR}

El arbitraje en el Ecuador se regula a través de la Ley de Arbitraje y Mediación $^{70}$ y por convenios internacionales ratificados, como la Convención de Nueva York..$^{71}$ Por otro lado, los arbitrajes también seguirán las directrices establecidas en los reglamentos de los centros de arbitraje a los que sometan su conflicto. Tras analizar estos cuerpos legales y algunos reglamentos de los centros de arbitraje más grandes de Ecuador, no se presenta restricción ni regulación alguna respecto del financiamiento por terceros en el arbitraje.

Por otro lado, se revisó también el Código Civil debido a que es la normativa que regula las relaciones entre privados, y a pesar de que no regula nada del TPF, admite una figura semejante; la cesión de derechos litigiosos. El Código Civil ecuatoriano establece,

Art. 1852.- Se cede un derecho litigioso cuando el objeto directo de la cesión es el evento incierto de la litis, del cual no se hace responsable el cedente. Se entiende litigioso un derecho, para los efectos de los siguientes artículos, desde que se cita judicialmente la demanda. ${ }^{72}$

69 Artículo 8.26: Investment. Comprehensive Economic And Trade Agreement (Ceta) Between Canada, Of The One Part, And The European Union And Its Member States, septiembre de 2017.

70 Ley de Arbitraje y Mediación [LAM], R.O. 417 de 14 de diciembre de 2006.

71 Convención sobre el reconocimiento y la ejecución de las sentencias arbitrales extranjeras [Convención de Nueva York], Nueva York, 10 de junio de 1958, ratificada por el Ecuador en 17 de diciembre de 1958. Reserva de Ecuador: Solamente se aplicará en lo referente a material comercial entendida según el derecho ecuatoriano.

72 Artículo 1852, Código Civil [CC], R.O. Suplemento 46 del 24 de junio de 2005, reformado por última vez R.O Suple- 
A pesar de que la cesión de derechos litigiosos no es lo mismo que el financiamiento por terceros en el arbitraje, es una figura similar que según lo establece Elina Mereminskaya, "Siguiendo la máxima jurídica qui potest plus, potest minus, la figura del TPFunding parece tener cabida plena". ${ }^{73}$ El principio jurídico qui potest plus, potest minus, lo explicó Jáñez diciendo que, "Consiste, entonces, en tener por ordenado o permitido de manera implícita, que se haga algo menor -de rango inferior- de lo que está ordenado o permitido expresamente por la ley. Quien puede lo más, puede lo menos". ${ }^{74}$

Lo que el trabajo establece con la comparación realizada entre el financiamiento por terceros y la cesión de derechos litigiosos, es que la legislación ecuatoriana permite una figura jurídica en la cual la parte poseedora del derecho de acción puede llegar al punto de vender por completo su derecho y dejar de ser parte dentro del proceso, y el tercero quien compró el derecho de acción, es quien pasaría a formar parte del proceso. Podría entenderse que este es el límite marcado por la Ley ecuatoriana: cualquier figura jurídica que sobrepase esto, ya no estaría permitida.

Por otro lado, el financiamiento por terceros es una figura en la cual la parte poseedora del derecho de acción solamente recibe financiamiento para los costos del proceso, abogado y demás gastos que sean necesarios para comenzar un arbitraje, pero sigue siendo la misma quien actúa dentro del proceso; el tercero financista no interviene dentro del mismo, solamente aporta con el capital. Figura que se entiende que está dentro del límite marcado por el Código Civil.

Entonces si la legislación ecuatoriana permite una figura jurídica en la cual la parte poseedora de un derecho de acción puede ceder este por completo a cambio de dinero; bajo el principio de que quien puede lo más puede lo menos y analizando ambas figuras bajo la misma legislación ¿por qué la parte poseedora del derecho no podría recibir dinero por parte de un tercero, sin la necesidad de ceder su derecho de acción por completo?, sino simplemente dar a cambio un porcentaje de la ganancia del laudo o sentencia que ponga fin al proceso.

Por lo que el contraste que el trabajo pretende señalar es que se podría entender que el financiamiento por terceros es una figura enmarcada dentro de los límites que establece la legislación ecuatoriana, ya que no vendría a ser una figura que va más allá o abusa del derecho de cesión de derechos litigiosos.

El Código Civil habla de venta o cesión de derechos, mientras que el TPF es un financiamiento; un préstamo para que la parte financiada acceda a la justi-

mento 96 de 08 de julio de 2019

73 Elina Mereminskaya, "Financiamiento de litigios a través de terceros y su aterrizaje en Chile", Informativo CAM N 14. Biblioteca Digital del Centro de Arbitraje y Mediación (CAM) de la Cámara de Comercio de Santiago (CCS), 2017.

74 Tarcisio Jánez Barrio, Lógica Jurídica, (Caracas: Editorial Universidad Católica Andrés Bello, 1999), 468. 
cia, pero sin la necesidad de renunciar a sus derechos litigiosos por completo; ${ }^{75}$ por lo que podría entenderse que el TPF es algo menor o de rango inferior a la cesión de derechos litigios. Por otro lado, también se debe tomar en cuenta el principio de autonomía de la voluntad, por el cual las partes pueden hacer todo aquello que no esté prohibido por la ley. ${ }^{76}$ Después de tomar en cuenta que: 1) los cuerpos normativos del Ecuador no regulan ni prohíben el TPF, 2) el Código Civil permite una figura similar y de mayor rango, y 3) basado en los principios qui potest plus, potest minus y autonomía de la voluntad; se podría deducir que el TPF no es contrario al orden público ecuatoriano.

\subsection{UNA ALTERNATIVA A LA REGULACIÓN ESTRICTA DEL TPF}

El TPF es un método de financiamiento que es criticado en la actualidad por su ausencia de regulación y los problemas que esta acarrea. ${ }^{77} \mathrm{Sin}$ embargo, existen posiciones contrarias, de que esta ausencia de regulación no necesariamente es perjudicial para el arbitraje y sus partes, sino que la libertad para actuar les ha permitido a las partes innovar con métodos como este y, a su vez, que se vean atraídos a implementarlo en sus arbitrajes. ${ }^{78}$

Si bien se evidenció que existen problemas como consecuencia del TPF no regulado y que son tantos los posibles escenarios que podrían llegar a ocurrir, que sería en extremo complicado poder tipificar todo de una forma estricta y concisa; sería difícil encasillar todo dentro de un ordenamiento jurídico. ${ }^{79}$

Este trabajo propone que los encargados de establecer reglas para estos arbitrajes financiados sean las instituciones en las que se administran los mismos. Así lo establecieron Marc Krestin y Rebecca Mulder: 'Las reglas de las instituciones tienen una aplicabilidad más amplia que las leyes nacionales y están diseñadas más específicamente para el proceso arbitral. Las directrices internacionales generalmente no son vinculantes y ofrecen una mayor flexibilidad". ${ }^{80}$

La propuesta no es una regulación estricta dentro del ordenamiento jurídico interno, sino guías básicas o principios generales, impuestos por los centros de arbitraje nacionales o internacionales, que sirvan para solucionar los conflictos que surjan en estos arbitrajes financiados. ${ }^{81}$

75 Morris y Ciambella, "Arbitration Funding in Singapore and Hong Kong".

76 Alfredo Bullard G., “¿Qué fue Primero el huevo o la gallina? El carácter contractual del recurso de anulación. ”. Revista Internacional de Arbitraje, )Julio-diciembre 2013)

77 Report of the Icca-Queen Mary Task Force On Third-Party Funding In International Arbitration.

78 Ver. Megan Betts y Evanthia Kasiora. "The Impact of the COVID-19 Pandemic on Third Party Funding and Security for Costs in International Commercial Arbitration". Kluwer Arbitration Blog. (Julio 2020). http://arbitrationblog.kluwerarbitration.com/2020/07/30/the-impact-of-the-covid-19-pandemic-on-third-party-funding-and-security-for-costs-in-international-commercial-arbitration/.

79 Krestin y Mulder. “TPF To Regulate Or Not?”.

80 Ibid.

81 Los arbitrajes ad hoc, podrían tomar estas recomendaciones de los centros en caso de desearlas, ya que los reglamentos son de conocimiento público. 
Establecer principios generales que sirvan como guía o recomendaciones es una propuesta aceptada por doctrinarios, por el Grupo de Trabajo de la ICCA, e incluso por Singapur, Hong Kong y Canadá, países que ya han regulado el TPF. Las recomendaciones serían guías clave que ayuden a las partes a tener mejores resultados en arbitrajes financiados, sin la necesidad de encasillarlos y limitarlos en reglas estrictas que les restrinjan actuar bajo el principio de autonomía de la voluntad, y sin el riesgo de que se regule más de lo necesario. Krestin y Mulder, han sustentado esta postura, señalando que:

Por lo tanto, propondríamos no optar por un sistema altamente fragmentado de leyes nacionales que regulen la financiación de terceros, sino desarrollar más un conjunto de directrices no vinculantes en las que los profesionales puedan confiar cuando se enfrenten a problemas de financiamiento de terceros en el arbitraje internacional..$^{82}$

Entre estas recomendaciones es fundamental el fomentar la comunicación entre las partes desde la primera etapa del arbitraje, e incitarlas a revelar el financiamiento y la identificación del financista, tanto a los árbitros como a la contraparte. ${ }^{83}$ El Grupo de Trabajo de la ICCA estableció que,

Los principios solo deben confirmar la autoridad de los árbitros e instituciones para solicitar la revelación de información, según sea necesario, y permitir que las partes y los financiadores hagan divulgaciones de hechos consideren relevantes para el conocimiento de los árbitros. ${ }^{84}$

Sin embargo, se respeta la posición de que el acuerdo de financiamiento debe ser privado entre la parte financiada y el tercero, por lo que no se debe obligar a revelar más allá de la identificación del financista. ${ }^{85} \mathrm{El}$ convenio de financiamiento es completamente ajeno al conflicto objeto de arbitraje, por lo que no tendría ninguna influencia en la litis. Francisco Blavi dice que,

La práctica sugiere que cualquier regulación de la industria de TPF debería centrarse en la divulgación de información relevante en lugar de crear un régimen sustantivo amplio. Definitivamente es innecesario exigir la divulgación completa de las condiciones de financiación en todos los arbitrajes internacionales. ${ }^{86}$

Este trabajo sugiere que el financiamiento por terceros podría funcionar en Ecuador sin regulación, y no llegaría a ser contrario al orden público, como se explicó en el capítulo anterior. El autor ecuatoriano, Andrés Larrea comenta al respecto que,

82 Krestin y Mulder. “TPF To Regulate Or Not?". (traducción no oficial).

83 Goldsmith y Melchionda, "Disclosure and Third-Party Funding".

84 Traducción Libre, Report of the Icca-Queen Mary Task Force On Third-Party Funding In International Arbitration, párr. 83.

85 Id., 189

86 Blavi. "It's time to regulate TPF". 
La legislación nacional no hace ninguna referencia a esta alternativa. Sin embargo, tampoco existe una norma legal que la prohíba, por lo tanto, una parte bien podría utilizar un third-party funder para financiar su próxima disputa judicial o arbitral. ${ }^{87}$

\subsubsection{EL TPF en BraSIL}

Brasil es uno de los países latinoamericanos en los que está creciendo la práctica del TPF, y a pesar de no tener una regulación al respecto en su ordenamiento jurídico, este país es un claro ejemplo de la teoría propuesta en el epígrafe anterior. El Centro de Arbitraje y Mediación de la Cámara de Comercio Brasil - Canadá, ${ }^{88}$ es el único centro de arbitraje que regula el TPF en Brasil, pero gracias a este centro la práctica de este método está creciendo en la nación suramericana. ${ }^{89}$

La Resolución 18/2016 de la CAM-CCBC, establece solamente recomendaciones con el objetivo de informar y orientar a las partes en los arbitrajes financiados. Es una resolución corta, de 5 artículos. Esta normativa comienza definiendo al TPF y definiendo los costos del procedimiento arbitral: costos administrativos, honorarios de abogados, árbitros y expertos o peritos; pero aclara que no se limita solamente a estos. ${ }^{90}$ De esta manera define el objeto de financiamiento, lo que el tercero aportará al arbitraje.

En sus siguientes artículos reconoce que el financiamiento por terceros podría acarrear problemas de conflictos de intereses que afecten la imparcialidad del árbitro, y recomienda que se informe a las partes lo antes posible del financiamiento y de la identificación completa del tercero que financiará sus costos. Una vez con la información revelada, las partes deberán verificar si existe o no un verdadero conflicto que pueda generar imparcialidad en el arbitraje. El tenor literal de la norma establece lo siguiente:

Artículo 4 - Con el fin de evitar posibles conflictos de interés, el CAM-CCBC recomienda a las partes que informen sobre la existencia de financiación de tercero en la primera oportunidad posible. En esta información, se debe incluir la identificación completa de la fuente de financiación.

Artículo 5 - Ante la información, el CAM-CCBC invitará a los árbitros a proceder a la verificación de conflicto y revelar cualquier hecho que pueda generar duda

87 Andrés Larrea. “:Es el Financiamiento por Terceros el Futuro de la Resolución de Disputas en Ecuador?”, Tobar ZVS SPINGARN. (Julio 2020). https://coronavirusresponse.tzvs.ec/es-el-financiamiento-por-terceros-el-futuro-de-la-resolucion-de-disputas-en-ecuador/

88 En adelante CAM-CCBC, por sus siglas.

89 Rodrigo M. Carneiro de Oliveira, Eider Avelino Silva and Rafael Curi Savastano, "The Third Party Litigation Funding Law Review: Brazil", The Law Review (2021).

90 Artículos 1 y 2, Resoluciones Administrativas RA 18/2016, Recomendaciones relativas a la existencia de financiación de tercero en arbitrajes administrados por el CAM-CCBC, aprobado el 01 de septiembre de 2011, con enmiendas de 28 de abril de 2016. 
justificada sobre su independencia e imparcialidad. La información acerca de la financiación de terceros también será proporcionada a la otra parte. ${ }^{91}$

Esta regulación del TPF por parte del CAM-CCBC es bastante similar a la regulación de los países revisados anteriormente. Se limita a establecer definiciones y recomendaciones, pero lo esencial de la regulación es que obliga la revelación del financiamiento y del financista, con el fin de evitar conflictos de intereses. No es una resolución que establezca normas estrictas, sino que señala recomendaciones para un mejor funcionamiento de arbitrajes financiados que sean administrados en su centro.

\section{4. ¿REgUlaR EL TPF o NO?}

Se han analizado los problemas y críticas a la ausencia de regulación del financiamiento por terceros, mismos que podrían llegar a presentarse en la práctica. Sin embargo, también se evidenció que no en todos los casos hubo inconvenientes. ${ }^{92}$ De este modo, la teoría planteada anteriormente busca promover el uso del TPF a la par de generar seguridad a las partes. "Es importante apoyar el desarrollo de TPF y aprovechar sus beneficios, al mismo tiempo que se regula lo necesario para evitar incertidumbres y limitar sus peligros". ${ }^{93}$

El trabajo propone que no es necesaria una regulación tan estricta dentro del ordenamiento de cada país, ni tampoco se necesitan instrumentos internacionales vinculantes para los países que quieran promover este método en los arbitrajes en su interior. ${ }^{94}$ La alternativa es promover recomendaciones generales respecto del funcionamiento de arbitrajes financiados, regulado por los reglamentos de los centros de arbitraje del Ecuador, y con esta regulación superar los problemas planteados previamente en este trabajo.

Entre los problemas a superar está el conflicto de intereses, en el cual enumerar la variedad de casos y de relaciones que podrían surgir entre árbitro, abogado y financista, sería sumamente complicado. ${ }^{95} \mathrm{La}$ recomendación ante este problema es establecer una obligación de revelación del financiamiento y la identidad del financista. Una vez revelada la identidad del tercero, se deberán revisar las Directrices IBA.

Como se planteó anteriormente en el trabajo, la revelación de toda la información relevante al TPF, permitirá a las partes analizar si la relación entre el

91 Artículos 4 y 5, Resoluciones Administrativas RA 18/2016, Recomendaciones relativas a la existencia de financiación de tercero en arbitrajes administrados por el CAM-CCBC, aprobado el 01 de septiembre de 2011, con enmiendas de 28 de abril de 2016.

92 Ver. Prior IAReporter Coverage Of Oxus Gold V. Uzbekistan. Se pueden revisar los reportes de este caso de la IA en https:// www.iareporter.com/arbitration-cases/oxus-gold-v-uzbekistan/.

93 Blavi, "It's time to regulate TPF".

94 Ver. Krestin y Mulder. "TPF To Regulate or Not?".

95 Ver. Krestin y Mulder. "TPF To Regulate or Not?”. 
financista y el árbitro entra en la categoría de no renunciable, ${ }^{96}$ obligando al árbitro a excusarse; o si entra en la categoría de renunciable, ${ }^{97}$ y las partes declaran su voluntad de que el árbitro continúe en el proceso a pesar de su relación con el financista; el árbitro podría continuar sin problema. En cualquiera de las dos situaciones, se eliminaría el conflicto de intereses, asegurando la imparcialidad del árbitro durante el resto del proceso.

Respecto al problema de las costas la propuesta no ahonda mucho al respecto dado que no es común que un tribunal acepte una solicitud de costas. ${ }^{98} \mathrm{El}$ grupo de trabajo de la ICCA-QM también estableció que "En ausencia de un poder expreso, en la legislación nacional aplicable o en las reglas procesales, un tribunal carecería de competencia para dictar una orden de costas contra un tercero financiador". ${ }^{99}$ Esto se debe a que si el tercero no es considerado parte del arbitraje, tampoco podría ser condenado bajo sus reglas. Al tercero solamente le es exigible aquello estipulado en su convenio. ${ }^{100}$

Sin embargo, la postura de este trabajo es que igual que enlistar la infinidad de posibles escenarios respecto al conflicto de intereses, sería muy difícil enumerar razones bajo las cuales sea obligatorio que un tribunal condene al pago de costas a una parte o a un financista. Además, alegar que la parte financiada tiene recursos suficientes para costear el arbitraje, no es una razón suficientemente fundamentada para comenzar a implementar una práctica de aprobar caución de costas contra financistas. Así lo señaló Carlos Ríos Pizarro,

No existe fundamento, en nuestra opinión, para verse obligado a condenar a una parte en costos por el mero hecho de existir un acuerdo de financiamiento. La condena en costos dependerá de una serie de factores como el comportamiento de las partes durante el arbitraje, las reglas procesales aplicables, la estimación de los árbitros e incluso el propio pacto de las partes. ${ }^{101}$

Respecto al problema de confidencialidad hacia el tercero financista, dependerá de las cláusulas bajo las cuales el financista y financiado manejen su acuerdo, y si la parte financiada le permitirá o no al tercero revisar el expediente de arbitraje. Dependerá de cada caso, dado que establecer un convenio de financiamiento estándar no es la solución; esto limitaría muchos financiamientos y haría que el TPF pierda su esencia y que no sea atractivo para aquellos que buscan invertir en arbitrajes. El informe de la ICCA-QM al respecto estableció que:

96 Listado Rojo Irrenunciable. Directrices IBA

97 Listado Rojo Renunciable, Listado Naranja y Listado Verde. Id.

98 Ríos, “TPF y su aplicación en arbitrajes comerciales.”

99 Traducción libre, Report of the Icca-Queen Mary Task Force On Third-Party Funding In International Arbitration. párr. 145 .

100 Traducción Libre, Id., 215-216.

101 Ríos, TPF y su aplicación en arbitrajes comerciales. 
El Grupo de Trabajo actúa con especial cuidado en sus propuestas para evitar una intromisión excesiva en la libertad de contratación de las partes, reconociendo que, siempre que las partes estén bien representadas, pueden celebrar una amplia gama de acuerdos. ${ }^{102}$

Sin embargo, la parte financiada tendrá la obligación de revelar su financiamiento y todo lo que este influya en el arbitraje, así lo han dicho Goldsmith y Melchionda, "Las instituciones deben adoptar una definición clara de TPF y requerir que las partes revelen cualquier relación cubierta por dicha definición". ${ }^{103}$ Por lo que, si las partes han acordado que el arbitraje será confidencial, tanto partes y árbitros deberán aclarar si esta confidencialidad imposibilita también al financista de revisar el expediente.

Otra crítica al TPF es que podría generar gran cantidad de demandas abusivas o partes que actúen de mala fe, abusando del derecho, presentando demandas en arbitraje solamente para retrasar procesos. ${ }^{104} \mathrm{Sin}$ embargo, no se considera un problema real, dado que los financistas realizan una debida diligencia respecto del caso, y no prestarían fondos a un caso que no tiene buenas oportunidades en las que él se vea beneficiado; ${ }^{105}$ el propósito tampoco es financiar cualquier caso que llegue a su puerta. Esto ya dependerá de la debida diligencia con la que actúen los financistas, ya que el arbitraje no debería entrar a regular el acuerdo de financiamiento.

\section{Conclusión}

Tras un profundo análisis a los problemas tanto doctrinarios como prácticos, se evidencia que la ausencia de regulación estricta no necesariamente acarreará problemas en todos los casos, y no se puede establecer una regla general. Con la teoría propuesta por este trabajo, la mayoría de los problemas planteados se ven superables sin la necesidad de regular el TPF de manera estricta; basta con ciertas recomendaciones a nivel general para brindar seguridad jurídica a las partes en un arbitraje financiado. ${ }^{106}$

Las partes deben ser libres de actuar bajo la autonomía de la voluntad, por lo que se debe evitar una regulación llena de barreras; ${ }^{107}$ misma que produciría que el arbitraje deje de ser un mercado atractivo para financistas, evitando que el TPF funcione. Si bien el arbitraje funciona muy bien sin este método, el

102 Traducción Libre, Report of the Icca-Queen Mary Task Force On Third-Party Funding In International Arbitration, párr. 186. (traducción no oficial).

103 Traducción Libre, Goldsmith y Melchionda, "Disclosure and Third-Party Funding”.

104 Stroble y Welikson, “TPF: Industry Developments".

105 "Ventajas Del Third Party Funding".

106 Krestin y Mulder. “TPF To Regulate Or Not?”.

107 Morris y Ciambella, "Arbitration Funding in Singapore and Hong Kong". 
TPF ayudaría a muchos interesados a acceder al arbitraje. ${ }^{108}$ Brindar acceso a la justicia es bueno tanto para los centros de arbitraje, como para la justicia ordinaria, ya que reduce la carga de casos a los jueces. ${ }^{109}$

La ausencia de regulación del TPF puede, incluso, ser la esencia para que este método funcione, ya que ante la falta de limitaciones, la parte financiada y el financista pueden pactar un acuerdo libremente de manera privada, con el fin de que obtengan un beneficio mutuo; llamando la atención a comerciantes y estudios jurídicos a invertir en arbitrajes financiados, y a las partes a recibir apoyo económico y disminuir los riesgos de costos. Woodsford, un equipo de financistas expertos, establecen que:

Los demandantes sofisticados aprecian cómo el financiamiento puede ayudarlos a administrar los costos y compensar el riesgo legal. Los abogados emprendedores reconocen cómo la financiación de terceros puede ayudarlos a expandir su práctica $\mathrm{y}$ ofrecer a los clientes flexibilidad en los honorarios. ${ }^{110}$

A pesar de que los problemas analizados son superables, el trabajo plantea a manera de conclusión y con el fin de dar un aporte a este tema, que los centros de arbitraje son las entidades más aptas para poder regular el TPF. Recaerá en cada tribunal y árbitro la responsabilidad de analizar al TPF en la práctica, para así poder dar parámetros a tomar en cuenta para la regulación de este método en las respectivas instituciones arbitrales.

Aterrizando la investigación en Ecuador, bajo el principio de autonomía de la voluntad y de que quien puede lo más puede lo menos; se concluyó que el TPF no es contrario al orden público en Ecuador. Brindando así una respuesta al problema jurídico del presente trabajo; estableciendo que la ausencia de regulación no acarrea consecuencias negativas para el arbitraje, pero que sí puede mejorarse su práctica a través de pequeñas recomendaciones dadas por los reglamentos de los centros de arbitraje.

Finalmente, el trabajo concluye con que no es necesaria una regulación estricta que establezca barreras o prohibiciones al TPF. La alternativa de regular a través de recomendaciones generales es suficiente para superar los problemas que han sido planteados por aquellos autores que critican el TPF. Con estas recomendaciones y principios, se crearán mejores costumbres dentro de la comunidad del arbitraje, al momento de trabajar con arbitrajes financiados

\footnotetext{
108 Kadhim, "Third Party Funding Issues in Arbitration".

109 Silvia Barona y Carlos Esplugues, "ADR Mechanism and They Incorporation into Global Justice in the Twenty-first Century: Some Concepts and Trends", Global Perspectives on ADR (2014)

110 Morris y Ciambella, "Arbitration Funding in Singapore and Hong Kong". (traducción no oficial).
} 


\section{RECOMENDACIONES}

Con el parámetro que establece este artículo académico, se espera que los centros de arbitraje en el Ecuador comiencen a regular el TPF en sus reglamentos internos, con el fin de llamar la atención de la comunidad de arbitraje, para que sepan que la legislación ecuatoriana es amigable con el TPF y que podría aumentarse crecer la práctica de arbitrajes financiados en el Ecuador, tanto a nivel doméstico como de arbitrajes internacionales que pudieren ejecutarse en Ecuador.

Se recomienda revisar la regulación del TPF en Hong Kong, Singapur y Canadá, como guías y ejemplos para la regulación en Ecuador. Incluso prestar una mayor atención a la Resolución 18/2016 de la CAM-CCBC, dado que es una realidad más cercana a la propuesta de este trabajo, ya que Brasil ha aumentado su práctica de arbitrajes financiados, solamente a través de regular el TPF en el reglamento interno de la CAM-CCBC. La experiencia de estos países será una gran guía para que los centros de arbitraje ecuatorianos establezcan una regulación efectiva sobre el TPF.

En la regulación, se considera necesario establecer la obligación de revelar toda la información relevante del financiamiento. No basta con revelar que existe un financiamiento, sino que se debe dar a conocer la identidad del tercero financista, para analizar a fondo cualquier posible relación entre el árbitro y el financista, con el fin de establecer, bajo las Directrices de la IBA, si existe o no un conflicto de intereses. No se considera necesario revelar más información que esta, dado que también hay que respetar la confidencialidad que caracteriza al contrato entre el tercero y la parte financiada.

Respecto al problema que surge de que no se puede tratar al financista como una parte del arbitraje y por lo tanto el árbitro no tiene potestad sobre él, la recomendación, apoyada por pocos doctrinarios dado que no se ha hablado mucho al respecto, es que se podría tratar al tercero financista bajo la figura de un tercero no signatario, de esta forma el mismo entraría a ser parte del arbitraje y el tribunal tendría potestad sobre él, habilitándose la posibilidad de condenar a costas tanto a la parte como a su financista. ${ }^{111}$ Postura muy compleja que amerita su propia investigación a fondo.

111 Juan Pablo Labbé Aroca, "La extensión del acuerdo de arbitraje a terceros aparentes en el Arbitraje Comercial Internacional: análisis de algunas teorías", Revista de Derecho 2 (2018), 201-236. 
